\title{
Gauss-Bonnet inflation with a constant rate of roll
}

\author{
Tie-Jun Gao ${ }^{\mathrm{a}}$ \\ School of Physics and Optoelectronic Engineering, Xidian University, Xi' an 710071, China
}

Received: 10 August 2020 / Accepted: 19 October 2020 / Published online: 2 November 2020

(C) The Author(s) 2020

\begin{abstract}
In the model of the inflaton nonminimal coupling to the Gauss-Bonnet term, we discuss the constant-roll inflation with constant $\epsilon_{1}$, constant $\epsilon_{2}$ and constant $\eta_{H}$, respectively, with the additional assumption that $\delta_{1}$ is a constant. Using the Bessel function approximation, we get the analytical expressions for the scalar and tensor power spectrum and derive the scalar spectral index $n_{\mathcal{R}}$ and the tensor to scalar ratio $r$ to the first order of $\epsilon_{1}$. By using the Planck 2018 observations constraint on $n_{\mathcal{R}}$ and $r$, we obtain some feasible parameter space and show the result on the $n_{\mathcal{R}}-r$ region. The scalar potential is also reconstructed in some special cases.
\end{abstract}

\section{Introduction}

Inflation in the early Universe has become a well established part of modern cosmology, it naturally generates the density perturbations that become the seeds of large-scale structure and temperature anisotropies of the cosmic microwave background (CMB). The predictions have been confirmed by numerous observations, such as the WMAP [1] and Planck space missions [2]. Recently, the Planck 2018 data constrain the scalar spectral index and the tensor-to-scalar ratio to be $n_{\mathcal{R}}=0.9649 \pm 0.0042$ at $68 \%$ confidence level and $r_{0.002}<0.064$ at $95 \%$ confidence level [2], which will narrow down some inflationary models.

In the conventional inflationary models, it is usually assumed that the scalar field rolls down slowly, or more precisely to assumed that the flow parameters satisfy the conditions $\epsilon_{H} \ll 1$ and $\eta_{H} \ll 1$. This so called slow-roll inflation leads to a nearly scale-invariant spectrum of density perturbations and is consistent with the latest observations. Recently, a new type of inflation called constant-roll inflation has been put forward in [3], where one of the flow parameters $\eta_{H}$ is assumed to be a constant, and not necessarily small. Such

a e-mail: tjgao@xidian.edu.cn (corresponding author) class of models can also produce a scale-invariant spectrum and is compatible with the observational constraints without the assumption of slow-roll approximation. The constantroll condition with small $\eta_{H}$ is compatible with the slow-roll inflation, and a special value $\eta_{H}=3$ amounts to the ultraslow-roll inflation $[4,5]$, in which the potential is very flat and the inflaton almost stops rolling. The constant-roll inflationary models are discussed in more details in [6-8], and extended to modified theories of gravity [9-14] or two fields case [15], and using other constant-roll conditions [16-18]. Other developments are appeared in [19-22].

Since the inflation occurs in the early Universe which is believed to be described by quantum gravity, so it is interest to discuss inflation in the framework of quantum gravity theories, such as string theory. It is known that when discuss the effective action in the early universe, the correction terms of higher orders in the curvature coming from superstrings may play a significant role, and the simplest of such correction is the Gauss-Bonnet (GB) term in the low-energy effective action of the heterotic string $[23,24]$. There are many works discussing accelerating cosmology with the GB correction in four and higher dimensions [25-28], and with nonminimal coupled to the inflaton [29-37] or to the Higgs [38-42].

In this work, we shall discuss the constant-roll inflation in the model with the inflation field $\phi$ nonminimal coupled to the GB term with one of the flow parameters, $\epsilon_{1}, \epsilon_{2}$ or $\eta_{H}$ is constant. The presence of such nonminimal coupling will generate a new degree of freedom, so here we consider an additional assumption that the first GB flow parameter $\delta_{1}$ is also a constant. We derive the analytical expressions of the scalar spectral index $n_{\mathcal{R}}$ and the tensor to scalar ratio $r$ for each case. Combine with the Planck 2018 observations, we obtain some feasible parameter spaces, and find that in some cases, the chosen of GB flow parameter $\delta_{1}>1$ can also produce a nearly scale-invariant scalar power spectrum, which is different from the slow-roll inflation. At last, we also reconstruct the potential in some spectral cases. 
The outline of this paper is as follows: in the next section, we briefly review the inflationary model with nonminimal coupling to the Gauss-Bonnet term. In Sections 3, 4 and 5, we focus on the inflationary models with constant $\epsilon_{1}$, constant $\epsilon_{2}$ or constant $\eta_{H}$, respectively, derive the formalism for the scalar spectral index and the tensor-to-scalar ratio of each model and constrain the parameter space with the Planck 2018 data. In Sect. 6, we try to reconstruct the potential in some special cases. And the final section is devoted to summary.

\section{Inflation with a Gauss-Bonnet coupling}

In this section, we shall review the inflation with the GB coupling, and present the mode equations of scalar and tensor perturbations. Consider the following action with the inflation field $\phi$ coupled to the GB term

$$
S=\int d^{4} x \sqrt{-g}\left[\frac{1}{2} R-\frac{\omega}{2}(\nabla \phi)^{2}-V(\phi)-\frac{1}{2} \xi(\phi) R_{\mathrm{GB}}^{2}\right],
$$

where $V(\phi)$ is the potential for the scalar field $\phi, R_{\mathrm{GB}}^{2}=$ $R_{\mu \nu \rho \sigma} R^{\mu \nu \rho \sigma}-4 R_{\mu \nu} R^{\mu \nu}+R^{2}$ is the GB term, and $\xi(\phi)$ is the coupling functional. The coefficient $\omega$ in the kinetic term can be chosen the values \pm 1 to ensure conventional inflationary behaviour $[29,30]$. We work in Planckian units, $\hbar=c=8 \pi G=1$.

In the Friedmann-Robertson-Walker homogeneous universe, the background equations can be written as

$$
\begin{aligned}
& 6 H^{2}=\omega \dot{\phi}^{2}+2 V+24 \dot{\xi} H^{3}, \\
& 2 \dot{H}=-\omega \dot{\phi}^{2}+4 \ddot{\xi} H^{2}+4 \dot{\xi} H\left(2 \dot{H}-H^{2}\right), \\
& \omega(\ddot{\phi}+3 H \dot{\phi})+V_{, \phi}+12 \xi, \phi H^{2}\left(\dot{H}+H^{2}\right)=0,
\end{aligned}
$$

where a dot represents a derivative with respect to cosmic time and $(\ldots)_{, \phi}$ denotes a derivative with respect to the field $\phi$.

In standard inflation, it is useful to define a series of flow parameters, such as the Hubble flow parameters

$$
\epsilon_{H}=-\frac{\dot{H}}{H^{2}}, \quad \eta_{H}=-\frac{\ddot{H}}{2 H \dot{H}},
$$

furthermore, we also introduce the horizon flow parameters

$$
\epsilon_{1}=-\frac{\dot{H}}{H^{2}}, \quad \epsilon_{i+1}=\frac{\dot{\epsilon}_{i}}{H \epsilon_{i}}, \quad i \geq 1 .
$$

In the presence of the GB coupling, the new degrees of freedom suggest to defining another hierarchy of flow parameters [30]

$$
\delta_{1}=4 \dot{\xi} H, \quad \delta_{i+1}=\frac{\dot{\delta}_{i}}{H \delta_{i}}, \quad i \geq 1 .
$$

For the slow-roll inflation, these flow parameters should satisfy the slow-roll conditions $\left|\epsilon_{i}\right| \ll 1$ and $\left|\delta_{i}\right| \ll 1$. However, in the constant-roll inflation, such conditions are not necessary to satisfy, we only need $0<\epsilon_{1}<1$ to achieve a significative inflation.

At linear order in perturbation theory, the Fourier modes of curvature perturbations satisfy the Mukhanov-Sasaki equation

$$
v^{\prime \prime}+\left(c_{\mathcal{R}}^{2} k^{2}-\frac{z_{\mathcal{R}}^{\prime \prime}}{z_{\mathcal{R}}}\right) v=0
$$

where a prime represents the derivative with respect to conformal time $\tau . z_{\mathcal{R}}$ and the sound speed $c_{\mathcal{R}}$ can be expressed in terms of the horizon and GB flow parameters as

$z_{\mathcal{R}}^{2}=a^{2} \frac{F}{\left(1-\frac{1}{2} \Delta\right)^{2}}$,

$c_{\mathcal{R}}^{2}=1-\Delta^{2} \frac{2 \epsilon_{1}+\frac{1}{2} \delta_{1}\left(1-5 \epsilon_{1}-\delta_{2}\right)}{F}$,

with $\Delta=\delta_{1} /\left(1-\delta_{1}\right)$ and $F \equiv 2 \epsilon_{1}-\delta_{1}\left(1+\epsilon_{1}-\delta_{2}\right)+$ $\frac{3}{2} \Delta \delta_{1}$. And the effective mass term reads

$$
\begin{aligned}
\frac{z_{\mathcal{R}}^{\prime \prime}}{z_{\mathcal{R}}}= & a^{2} H^{2}\left[2-\epsilon_{1}+\frac{3}{2} \frac{\dot{F}}{H F}+\frac{3}{2} \frac{\dot{\Delta}}{H\left(1-\frac{1}{2} \Delta\right)}+\frac{1}{2} \frac{\ddot{F}}{H^{2} F}\right. \\
& +\frac{1}{2} \frac{\ddot{\Delta}}{H^{2}\left(1-\frac{1}{2} \Delta\right)}-\frac{1}{4} \frac{\dot{F}^{2}}{H^{2} F^{2}}+\frac{1}{2} \frac{\dot{\Delta}^{2}}{H^{2}\left(1-\frac{1}{2} \Delta\right)^{2}} \\
& \left.+\frac{1}{2} \frac{\dot{\Delta}}{H\left(1-\frac{1}{2} \Delta\right)} \frac{\dot{F}}{H F}\right],
\end{aligned}
$$

with

$$
\begin{aligned}
\frac{\dot{F}}{H}= & \epsilon_{1} \epsilon_{2}\left(2-\delta_{1}\right)-\delta_{1} \delta_{2}\left(1+\epsilon_{1}-\delta_{2}-\delta_{3}\right) \\
& +\frac{3}{2} \Delta \delta_{2}\left(\Delta+\delta_{1}\right), \\
\frac{\dot{\Delta}}{H}= & \Delta^{2} \frac{\delta_{2}}{\delta_{1}}, \\
\ddot{H}= & \epsilon_{1} \epsilon_{2}\left(-\epsilon_{1}+\epsilon_{2}+\epsilon_{3}\right)\left(2-\delta_{1}\right) \\
& +\epsilon_{1} \delta_{1} \delta_{2}\left(1+\epsilon_{1}-2 \epsilon_{2}-\delta_{2}-\delta_{3}\right) \\
& -\delta_{1} \delta_{2}^{2}\left(1+\epsilon_{1}-\delta_{2}-\delta_{3}\right) \\
& -\delta_{1} \delta_{2} \delta_{3}\left(1+\epsilon_{1}-2 \delta_{2}-\delta_{3}-\delta_{4}\right) \\
& +\frac{3}{2} \Delta \delta_{2}\left(\Delta+\delta_{1}\right)\left(-\epsilon_{1}+\Delta \frac{\delta_{2}}{\delta_{1}}+\delta_{3}\right) \\
& +\frac{3}{2} \Delta \delta_{2}\left(\Delta^{2} \frac{\delta_{2}}{\delta_{1}}+\delta_{1} \delta_{2}\right), \\
\ddot{\Delta}= & \Delta^{2} \frac{\delta_{2}}{\delta_{1}}\left(-\epsilon_{1}+2 \Delta \frac{\delta_{2}}{\delta_{1}}-\delta_{2}+\delta_{3}\right) .
\end{aligned}
$$


Similarly, for the tensor perturbations, the Fourier modes satisfy

$$
u^{\prime \prime}+\left(c_{T}^{2} k^{2}-\frac{z_{T}^{\prime \prime}}{z_{T}}\right) u=0,
$$

with

$$
\begin{aligned}
& z_{T}^{2}=a^{2}\left(1-\delta_{1}\right), \\
& c_{T}^{2}=1+\Delta\left(1-\epsilon_{1}-\delta_{2}\right) .
\end{aligned}
$$

And the effective mass term in the tensor mode can be written in terms of the flow parameters

$$
\frac{z_{T}^{\prime \prime}}{z_{T}}=a^{2} H^{2}\left[2-\epsilon_{1}-\frac{3}{2} \Delta \delta_{2}-\frac{1}{2} \Delta \delta_{2}\left(-\epsilon_{1}+\delta_{2}+\delta_{3}\right)-\frac{1}{4} \Delta^{2} \delta_{2}^{2}\right] .
$$

In the following sections, we shall focus on the constant-roll inflation in the model with nonminimal coupling to the GB term.

\section{Constant-roll inflation with constant $\epsilon_{1}$}

We focus on the constant-roll inflation in the model with nonminimal coupling to the GB term. The presence of such coupling will generate a new degree of freedom $\xi(\phi)$, so in this work we consider an additional assumption that the first GB flow parameter $\delta_{1}$ is also a constant, then from the definition of GB flow parameters (7) we have $\delta_{i}=0(i \geq 2)$.

In the following, we first discuss the case with $\epsilon_{1}=$ constant. From the relations

$$
\frac{d}{d \tau}\left(\frac{1}{a H}\right)=-1+\epsilon_{1}
$$

and assuming that $\epsilon_{1}$ is a constant, one can express the factor $a H$ in (11) and (16) as a function of conformal time $\tau$

$a H=-\frac{1}{\tau}\left(\frac{1}{1-\epsilon_{1}}\right)$,

then

$v_{\mathcal{R}}^{2}=\tau^{2} \frac{z_{\mathcal{R}}^{\prime \prime}}{z_{\mathcal{R}}}+\frac{1}{4}$,

can be approximated as a constant. Therefore the general solution to the mode equation (8) is a linear combination of Hankel functions of order $v_{\mathcal{R}}$

$$
v=\frac{\sqrt{\pi|\tau|}}{2} e^{i\left(1+2 v_{\mathcal{R}}\right) \pi / 4}\left[c_{1} H_{v_{\mathcal{R}}}^{(1)}\left(c_{\mathcal{R}} k|\tau|\right)+c_{2} H_{v_{\mathcal{R}}}^{(2)}\left(c_{\mathcal{R}} k|\tau|\right)\right]
$$

Choosing $c_{1}=1$ and $c_{2}=0$, the usual Minkowski vacuum state is recovered in the asymptotic past. Then on superhorizon scales, $c_{\mathcal{R}} k \ll a H$, the power spectrum of the scalar perturbation is

$$
\begin{aligned}
\mathcal{P}_{\mathcal{R}}= & \frac{c_{\mathcal{R}}^{-3}}{F} \frac{H^{2}}{4 \pi^{2}}\left(\frac{1-\Delta / 2}{a H|\tau|}\right)^{2} \frac{\Gamma^{2}\left(v_{\mathcal{R}}\right)}{\Gamma^{2}(3 / 2)}\left(\frac{c_{\mathcal{R}} k|\tau|}{2}\right)^{3-2 v_{\mathcal{R}}} \\
& \simeq \frac{2^{2 v_{\mathcal{R}}-3} c_{\mathcal{R}}^{-3}}{F} \frac{H^{2}}{4 \pi^{2}} \frac{\Gamma^{2}\left(v_{\mathcal{R}}\right)}{\Gamma^{2}(3 / 2)}\left(1-\frac{\Delta}{2}\right)^{2} \\
& \times\left.\left(\frac{1}{1-\epsilon_{1}}\right)^{1-2 v_{\mathcal{R}}}\right|_{c_{\mathcal{R}} k=a H}
\end{aligned}
$$

with the scalar spectral index is

$n_{\mathcal{R}}-1=\frac{d \ln \mathcal{P}_{\mathcal{R}}}{d \ln k}=3-2 \nu_{\mathcal{R}}$.

Using the same procedure as in the case of scalar perturbations, we get the power spectrum of tensor perturbations

$$
\begin{aligned}
\mathcal{P}_{T}= & \frac{8 c_{T}^{-3}}{1-\delta_{1}} \frac{H^{2}}{4 \pi^{2}}\left(\frac{1}{a H|\tau|}\right)^{2} \frac{\Gamma^{2}\left(v_{T}\right)}{\Gamma^{2}(3 / 2)}\left(\frac{c_{T} k|\tau|}{2}\right)^{3-2 v_{T}} \\
& \left.\simeq 2^{2 v_{T}} c_{T}^{-3} \frac{H^{2}}{4 \pi^{2}} \frac{\Gamma^{2}\left(v_{T}\right)}{\Gamma^{2}(3 / 2)}\left(\frac{1}{1-\delta_{1}}\right)\left(\frac{1}{1-\epsilon_{1}}\right)^{1-2 v_{T}}\right|_{c_{T} k=a H},
\end{aligned}
$$

with

$v_{T}^{2}=\tau^{2} \frac{z_{T}^{\prime \prime}}{z_{T}}+\frac{1}{4}$,

and the tensor spectral index is

$n_{T}=\frac{d \ln \mathcal{P}_{T}}{d \ln k}=3-2 v_{T}$.

Combing Eqs. (21) and (23), we obtain the tensor to scalar ratio

$r \equiv \frac{\mathcal{P}_{T}}{\mathcal{P}_{\mathcal{R}}} \simeq 2^{3+2 v_{T}-2 v_{\mathcal{R}}} F \frac{c_{\mathcal{R}}^{3}}{c_{T}^{3}} \frac{\Gamma^{2}\left(v_{T}\right)}{\Gamma^{2}\left(v_{\mathcal{R}}\right)} \frac{\left(1-\epsilon_{1}\right)^{2 v_{T}-2 v_{\mathcal{R}}}}{(1-\Delta / 2)^{2}\left(1-\delta_{1}\right)}$.

Below we will assume that the first GB flow parameter $\delta_{1}$ is a constant, which contains two cases: $\delta_{1}=0$ or $\delta_{1} \neq 0$.

\section{$3.1 \delta_{1}=0$}

If $\delta_{1}=0$, using (22), (26) we get

$$
\begin{aligned}
& n_{\mathcal{R}}=4-\left|\frac{3-\epsilon_{1}}{1-\epsilon_{1}}\right|, \\
& r=16 \epsilon_{1} .
\end{aligned}
$$




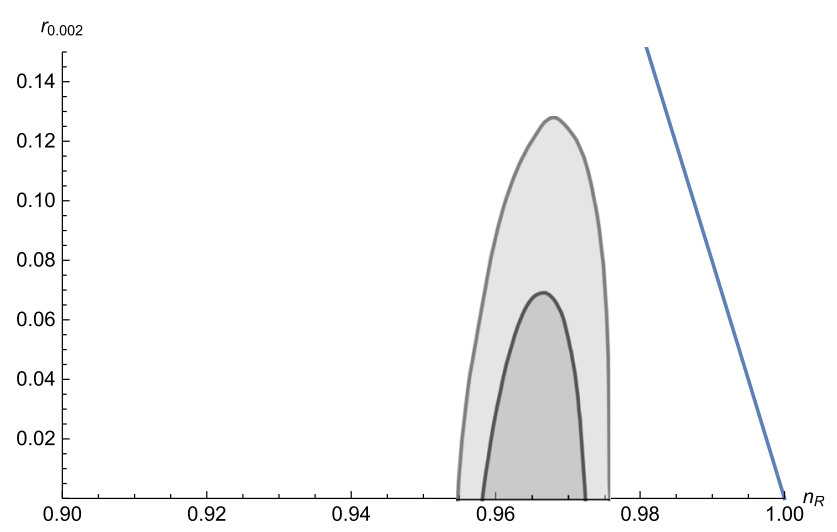

Fig. 1 The $n_{\mathcal{R}}-r$ region predicted by the model with $\epsilon_{1}=$ constant and $\delta_{1}=0$. The contours are the marginalized joint $68 \%$ and $95 \%$ confidence level regions for $n_{\mathcal{R}}$ and $r$ at the pivot scale $k_{*}=0.002$ $\mathrm{Mpc}^{-1}$ from the Planck $2018 \mathrm{TT}, \mathrm{TE}, \mathrm{EE}+$ lowE + lensing data [2]

The $n_{\mathcal{R}}-r$ region predicted by the model with $\epsilon_{1}=$ constant and $\delta_{1}=0$ are show in Fig. 1, where the contours are the marginalized joint $68 \%$ and $95 \%$ confidence level regions for $n_{\mathcal{R}}$ and $r$ at the pivot scale $k_{*}=0.002 \mathrm{Mpc}^{-1}$ from the Planck 2018 TT, TE, EE + lowE + lensing data.

We could see that the curve is ruled out by the observations, so we didn't interest in this case.

\section{$3.2 \delta_{1} \neq 0$}

If $\delta_{1}=$ constant but not zero, the expression of scalar spectral index is the same as in the previous case

$n_{\mathcal{R}}=4-\left|\frac{3-\epsilon_{1}}{1-\epsilon_{1}}\right|$

and the tensor-to-scalar ratio is more complex

$$
\begin{aligned}
r= & \frac{\left(16\left(\left(2 \delta_{1}^{2}-6 \delta_{1}+4\right) \epsilon_{1}+5 \delta_{1}^{2}-2 \delta_{1}\right)\right)}{\left(2-3 \delta_{1}\right)^{2}} \\
& \times\left(\frac{\left(3 \delta_{1}-2\right)\left(\left(\delta_{1}^{2}+2 \delta_{1}-2\right) \epsilon_{1}-2 \delta_{1}^{2}+\delta_{1}\right)}{\left(1-\delta_{1} \epsilon_{1}\right)\left(\left(2 \delta_{1}^{2}-6 \delta_{1}+4\right) \epsilon_{1}+5 \delta_{1}^{2}-2 \delta_{1}\right)}\right)^{3 / 2}
\end{aligned}
$$

Combine these expressions with the observational constraints from Planck 2018, one can obtain the constraints on $\epsilon_{1}$ and $\delta_{1}$. However, for a significative inflation we have $\ddot{a}>0$, which is equivalent to $\epsilon_{1}<1$, so only the parameter region satisfies $\epsilon_{1}<1$ is reasonable. We then obtain two regions of parameter space, and show them in Fig. 2.

In Fig. 3, we show the $n_{\mathcal{R}}-r$ region predicted by the model for the parameters in region I of Fig. 2 .

Left panel: The parameter $\delta_{1}$ is taken as $\delta_{1}=0.04,0.03$, 0.02 from left to right, and as $\epsilon_{1}$ increase, the $n_{\mathcal{R}}-r$ dots go along the curves to the left. Right panel: The parameter $\epsilon_{1}$ is taken as $\epsilon_{1}=0.02,0.015,0.01$ from left to right, and as $\delta_{1}$ increase, the $n_{\mathcal{R}}-r$ dots go along the curves from top to bottom. The $n_{\mathcal{R}}-r$ region for the parameters in region II of Fig. 2 are shown In Fig. 4.

Left panel: The parameter $\delta_{1}$ is taken as $\delta_{1}=0.49,0.5,0.51$ from left to right, and as $\epsilon_{1}$ increase, the $n_{\mathcal{R}}-r$ dots go along the curves to the left. Right panel: The parameter $\epsilon_{1}$ is taken as $\epsilon_{1}=0.02,0.015,0.01$ from left to right. We could see that this panel is almost the same as the one in Fig. 3, that is because the scalar spectral index depends only on $\epsilon_{1}$, so for the same chosen of $\epsilon_{1}$, the curves look the same. However, different from that panel, in this time the $n_{\mathcal{R}}-r$ dots go along the curves from bottom to top as $\delta_{1}$ increase.

We could see that for the choices of parameter space in Fig. 2, the inflation prediction is in agreement with the observational constraints. However, the constant-roll inflation with $\epsilon_{1}=$ constant is no natural end, hence an additional mechanism is required to stop it.

\section{Constant-roll inflation with constant $\epsilon_{2}$}

In this section, we assume that the second horizon flow parameter $\epsilon_{2}$ is a constant. So from the relations (17) and using the definition of flow parameter (6), we obtain that to the first order approximation of $\epsilon_{1}$, the relation between $a H$ and $\tau$ is $[16,18]$

$$
a H \simeq-\frac{1}{\tau}\left(1+\frac{\epsilon_{1}}{1-\epsilon_{2}}\right),
$$

then, we obtain the power spectrum of the scalar perturbation and tensor perturbation

$$
\begin{aligned}
\mathcal{P}_{\mathcal{R}} \simeq & \frac{2^{2 v_{\mathcal{R}}-3} c_{\mathcal{R}}^{-3}}{F} \frac{H^{2}}{4 \pi^{2}} \frac{\Gamma^{2}\left(v_{\mathcal{R}}\right)}{\Gamma^{2}(3 / 2)}\left(1-\frac{\Delta}{2}\right)^{2} \\
& \times\left.\left(1+\frac{\epsilon_{1}}{1-\epsilon_{2}}\right)^{1-2 v_{\mathcal{R}}}\right|_{c_{\mathcal{R}} k=a H}, \\
\mathcal{P}_{T} \simeq & 2^{2 v_{T}} c_{T}^{-3} \frac{H^{2}}{4 \pi^{2}} \frac{\Gamma^{2}\left(v_{T}\right)}{\Gamma^{2}(3 / 2)}\left(\frac{1}{1-\delta_{1}}\right) \\
& \times\left.\left(1+\frac{\epsilon_{1}}{1-\epsilon_{2}}\right)^{1-2 v_{T}}\right|_{c_{T} k=a H},
\end{aligned}
$$

with the scalar spectral index and the tensor-to-scalar ratio are

$$
n_{\mathcal{R}}-1=\frac{d \ln \mathcal{P}_{\mathcal{R}}}{d \ln k}=3-2 v_{\mathcal{R}}
$$




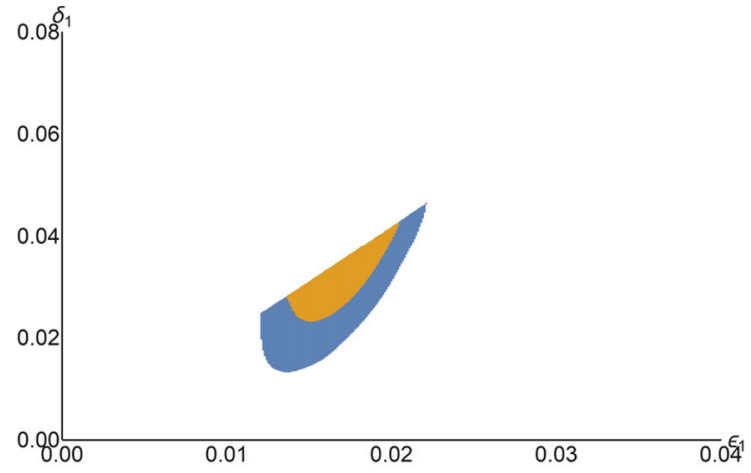

Region I

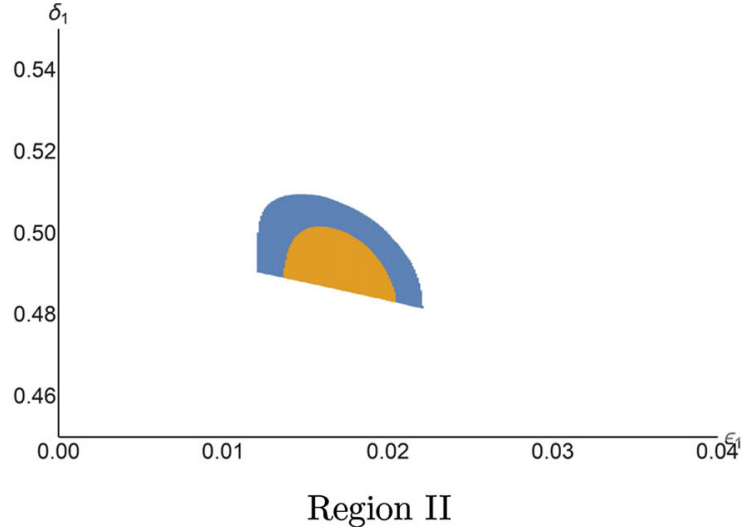

Region II

Fig. 2 The observational constraints on $\epsilon_{1}$ and $\delta_{1}$. The orange and blue regions correspond to the parameters satisfied $1 \sigma$ and $2 \sigma$ confidence level, respectively
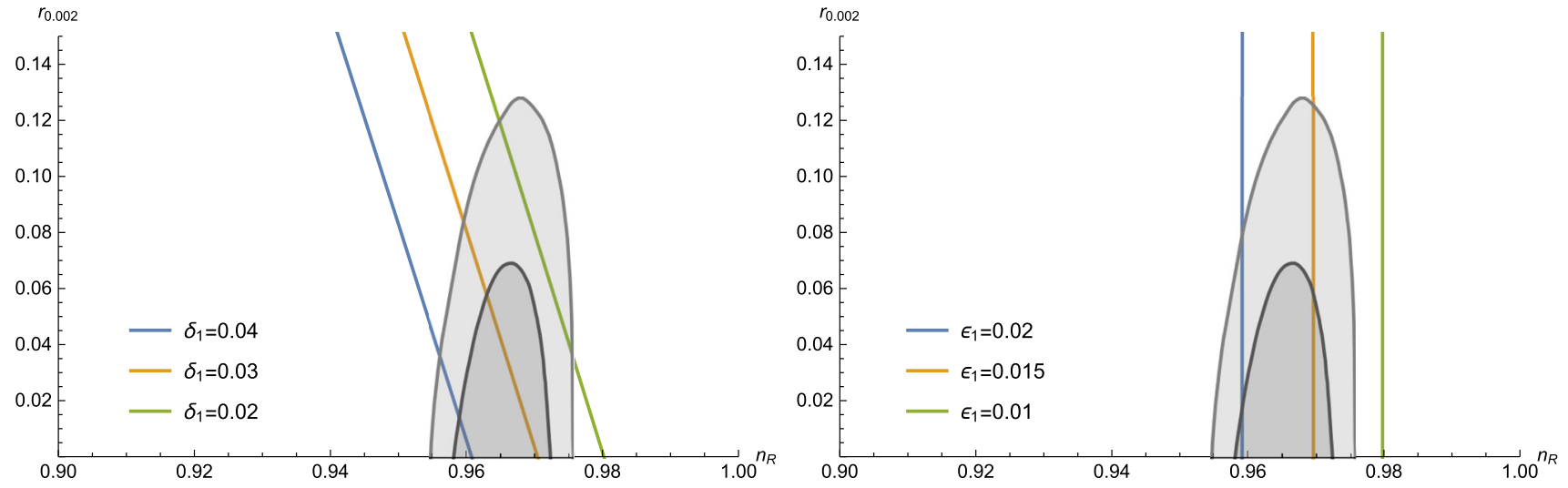

Fig. 3 The $n_{\mathcal{R}}-r$ region predicted by the model for the parameter region I of Fig. 2
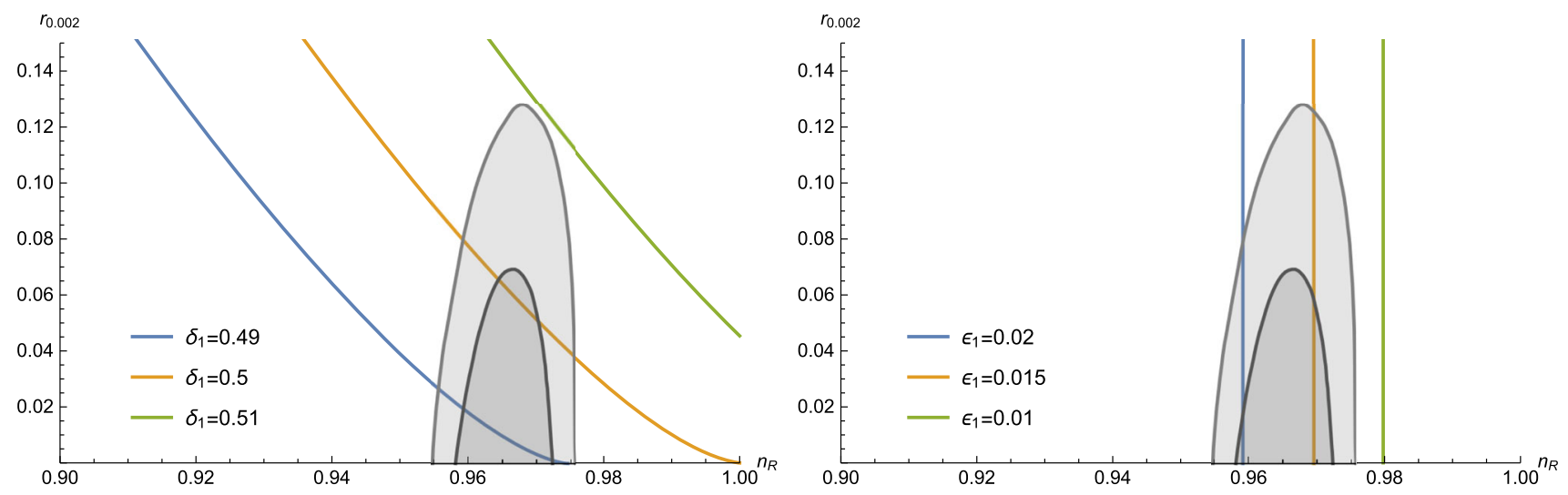

Fig. 4 The $n_{\mathcal{R}}-r$ region predicted by the model for the parameter region II of Fig. 2

$$
r \equiv \frac{\mathcal{P}_{T}}{\mathcal{P}_{\mathcal{R}}} \simeq 2^{3+2 v_{T}-2 v_{\mathcal{R}}} F \frac{c_{\mathcal{R}}^{3}}{c_{T}^{3}} \frac{\Gamma^{2}\left(v_{T}\right)}{\Gamma^{2}\left(v_{\mathcal{R}}\right)} \frac{\left(1+\frac{\epsilon_{1}}{1-\epsilon_{2}}\right)^{2 v_{\mathcal{R}}-2 v_{T}}}{(1-\Delta / 2)^{2}\left(1-\delta_{1}\right)} .
$$

In the following, we still assume that the first GB flow parameter $\delta_{1}$ is a constant, which contains two cases: $\delta_{1}=0$ or $\delta \neq 0$.
$4.1 \delta_{1}=0$

If $\delta_{1}=0$, the model recovers to the case without GB coupling, and such case have been discussed in other works [16]. Here we list some useful results. 


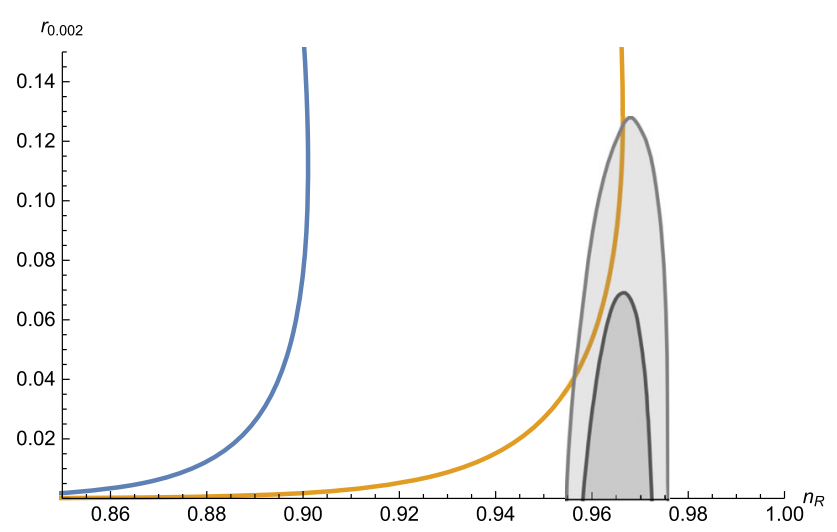

Fig. 5 The $n_{\mathcal{R}}-r$ region predicted by the model with $\delta_{1}=0$ and constant $\epsilon_{1}$ (blue line) or constant $\eta_{H}$ (orange line)

To the first order approximation of $\epsilon_{1}$, the scalar spectral index can be approximated as

$n_{\mathcal{R}} \simeq 4-\left|\epsilon_{2}+3\right|+\frac{\left(2 \epsilon_{2}^{2}+7 \epsilon_{2}+6\right) \epsilon_{1}}{\left(\epsilon_{2}-1\right)\left|\epsilon_{2}+3\right|}$

and the tensor-to-scalar ratio

$r \simeq 2^{3-\left|3+\epsilon_{2}\right|}\left(\frac{\Gamma[3 / 2]}{\Gamma\left[\left|3+\epsilon_{2}\right| / 2\right]}\right)^{2} 16 \epsilon_{1}$.

Since $\epsilon_{2}$ is a constant, we can integer the definition (6) and get the relation between $\epsilon_{1}$ and $\epsilon_{2}$

$\epsilon_{1}(N)=\exp \left(-\epsilon_{2} N\right)$

where $N$ is the e-folding number before the end of inflation, and we have use the condition $\epsilon_{1}(N=0)=1$ at the end of inflation. The $n_{\mathcal{R}}-r$ predictions compare with the Planck 2018 data are show in Fig. 5 (blue line) with $N=60$. We can see that the result is ruled out by the observations. So it is always to assume that the second Hubble flow parameter $\eta_{H}$ is a constant in the constant-roll inflationary models, we will discuss this case in the next section.

\section{$4.2 \delta_{1} \neq 0$}

If $\delta_{1}=$ constant but not zero, to the first order of $\epsilon_{1}$, the expression of scalar spectral index is and the tensor-to-scalar ratio

$$
\begin{aligned}
r \simeq & -\frac{16}{3\left(3 \delta_{1}-2\right)\left(5 \delta_{1}-2\right)} \sqrt{\frac{2-7 \delta_{1}+6 \delta_{1}^{2}}{2-5 \delta_{1}}}\left(30 \delta_{1}^{3}-27 \delta_{1}^{2}+6 \delta_{1}\right. \\
& +\epsilon_{1}\left(45 \delta_{1}^{4}-69 \delta_{1}^{3}-6 \delta_{1}^{2}+42 \delta_{1}-12\right. \\
& +\left(-2 \delta_{1}^{3}+7 \delta_{1}^{2}-7 \delta_{1}+2\right) \epsilon_{2}\left(\epsilon_{2}+3\right) \ln 4 \\
& \left.\left.+2\left(2 \delta_{1}^{3}-7 \delta_{1}^{2}+7 \delta_{1}-2\right) \epsilon_{2}\left(\epsilon_{2}+3\right)(\gamma-2+\ln 4)\right)\right),
\end{aligned}
$$

with $\gamma \approx 0.577216$ is the Euler-Mascheroni constant. Combine these expressions with the observational constraints and adopting the relation $\epsilon_{1}(N)=\exp \left(-\epsilon_{2} N\right)$, one can obtain the constraints on $\epsilon_{1}$ and $\delta_{1}$. By setting the e-folding number $N=60$, we find three regions of parameter space, and show them in Fig. 6.

The observational constraint on $n_{\mathcal{R}}-r$ for the parameters in region I of Fig. 6 are show in Fig. 7. Left panel: The parameter $\delta_{1}$ is taken as $\delta_{1}=-0.01$, $-0.006,-0.002$ from top to bottom, and as $\epsilon_{1}$ increase, the $n_{\mathcal{R}}-r$ dots go along the curves from left to right. Right panel: The parameter $\epsilon_{1}$ is taken as $\epsilon_{1}=0.12,0.16,0.2$ from left to right, and as $\delta_{1}$ increase, the $n_{\mathcal{R}}-r$ dots go along the curves to the left.

Similarly, in Fig. 8, we show the $n_{\mathcal{R}}-r$ region predicted for the parameters in region II of Fig. 6. Left panel: The parameter $\delta_{1}$ is taken as $\delta_{1}=0.1,0.09,0.08$ from left to right, and as $\epsilon_{1}$ increase, the $n_{\mathcal{R}}-r$ dots go along the curves from top to bottom. Right panel: $\epsilon_{1}$ is taken as $\epsilon_{1}=0.05,0.052,0.054$ from left to right, and as $\delta_{1}$ increase, the dots go along the curves from top to bottom.

Finally, the $n_{\mathcal{R}}-r$ region for the parameters in region III of Fig. 6 are show in Fig. 9. With $\delta_{1}$ is taken as $\delta_{1}=$ $0.505,0.503,0.501$ from left to right in the left panel, and as $\epsilon_{1}$ increase, the $n_{\mathcal{R}}-r$ dots go along the curves to the right. In the right panel, $\epsilon_{1}$ is taken as $\epsilon_{1}=0.065,0.07,0.075$ from left to right, and the dots go along the curves from bottom to top as $\delta_{1}$ increase.

\section{Constant-roll inflation with constant $\eta_{H}$}

In this section, the second Hubble flow parameter $\eta_{H}=$ $-\ddot{H} /(2 H \dot{H})$ is assumed to be a constant. Combine with the

$n_{\mathcal{R}} \simeq 1-\frac{2\left(3+\epsilon_{2}\right)\left(-2 \epsilon_{2}+2 \epsilon_{2}^{2}+\delta_{1}\left(2+3 \epsilon_{2}-3 \epsilon_{2}^{2}\right)+\delta_{1}^{2}\left(-5-\epsilon_{2}+\epsilon_{2}^{2}\right)\right)}{3\left(2 \delta_{1}-5 \delta_{1}^{2}\right)\left(1-\epsilon_{2}\right)} \epsilon_{1}$, 


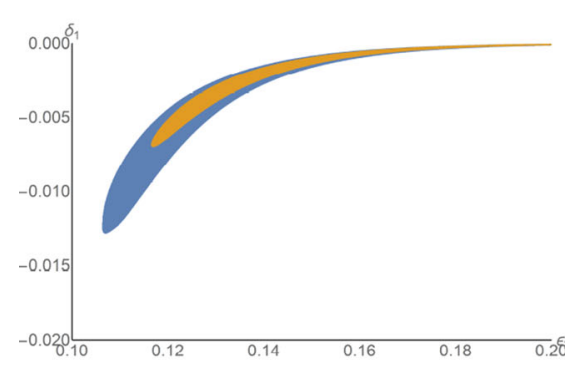

Region I

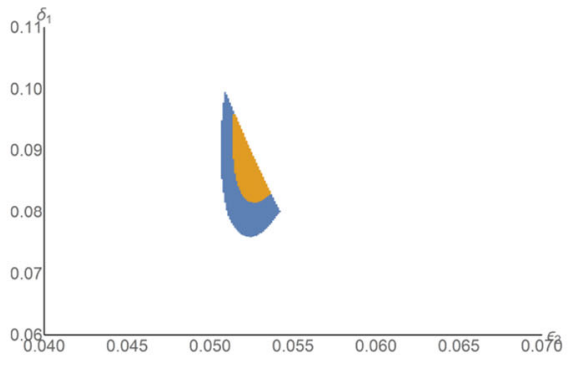

Region II

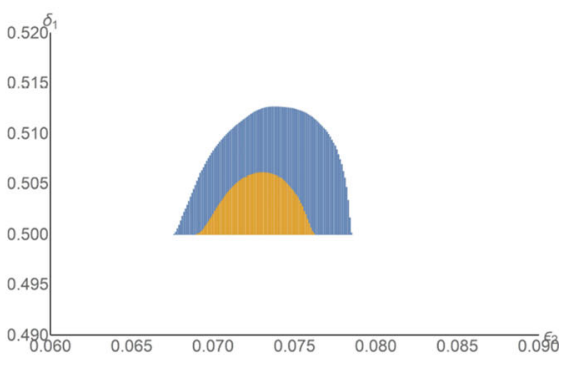

Region III

Fig. 6 The observational constraints on $\epsilon_{2}$ and $\delta_{1}$ with the e-folding number $N=60$. The orange and blue regions correspond to the parameters satisfied $1 \sigma$ and $2 \sigma$ confidence level, respectively
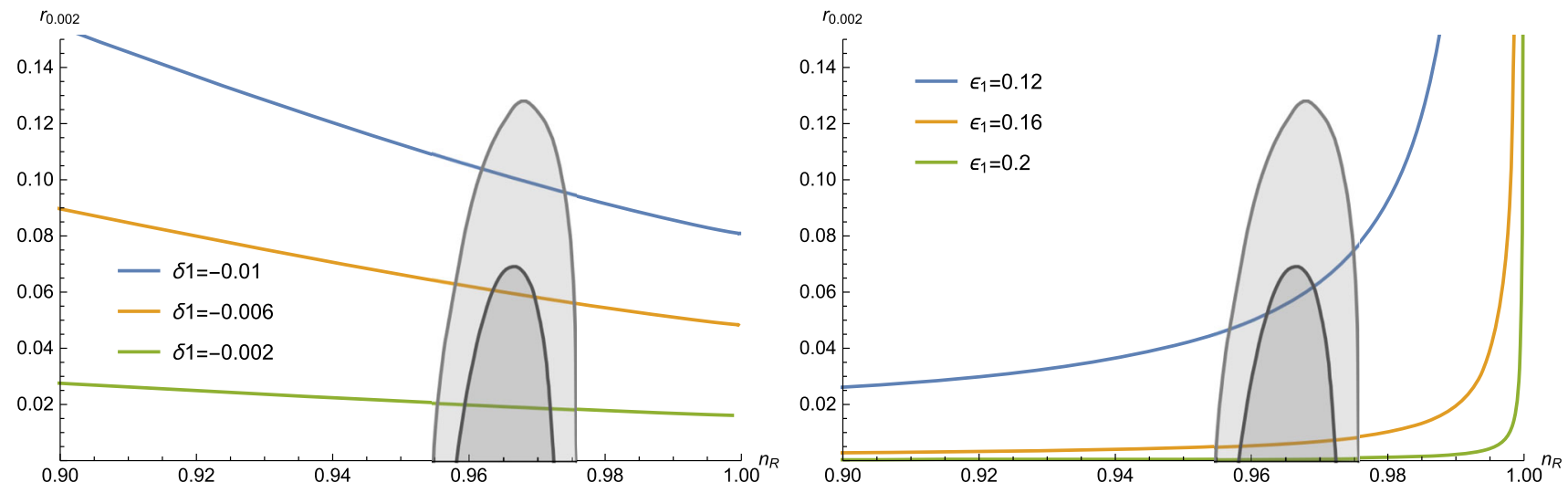

Fig. 7 The $n_{\mathcal{R}}-r$ region predicted by the model for the parameter region I of Fig. 6
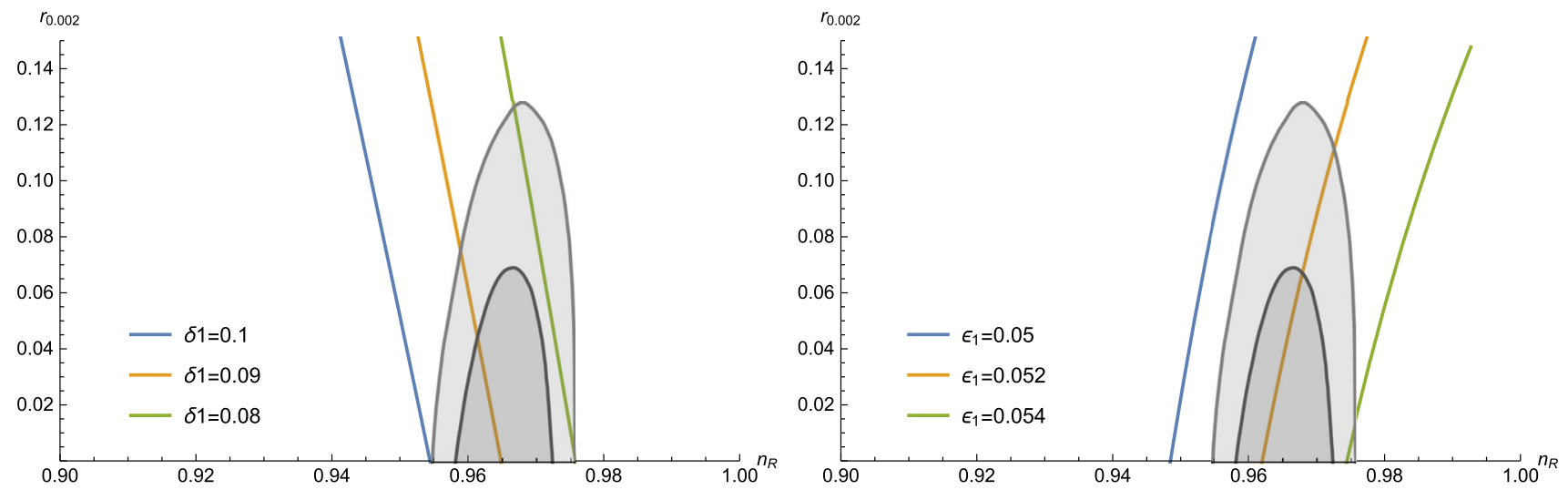

Fig. 8 The $n_{\mathcal{R}}-r$ region predicted by the model for the parameter region II of Fig. 6

Eq.(17), the relation between $a H$ and $\tau$ to the first order approximation of $\epsilon_{1}$ can be obtained as [18]

$a H \approx-\frac{1}{\tau}\left(1+\frac{\epsilon_{1}}{1+2 \eta_{H}}\right)$

Substitute this relation to the expressions of power spectrum, we get

$\mathcal{P}_{\mathcal{R}} \simeq \frac{2^{2 v_{\mathcal{R}}-3} c_{\mathcal{R}}^{-3}}{F} \frac{H^{2}}{4 \pi^{2}} \frac{\Gamma^{2}\left(v_{\mathcal{R}}\right)}{\Gamma^{2}(3 / 2)}\left(1-\frac{\Delta}{2}\right)^{2}$

$$
\begin{aligned}
& \times\left.\left(1+\frac{\epsilon_{1}}{1+2 \eta_{H}}\right)^{1-2 v_{\mathcal{R}}}\right|_{c_{\mathcal{R}} k=a H}, \\
\mathcal{P}_{T} \simeq & 2^{2 v_{T}} c_{T}^{-3} \frac{H^{2}}{4 \pi^{2}} \frac{\Gamma^{2}\left(v_{T}\right)}{\Gamma^{2}(3 / 2)}\left(\frac{1}{1-\delta_{1}}\right) \\
& \times\left.\left(1+\frac{\epsilon_{1}}{1+2 \eta_{H}}\right)^{1-2 v_{T}}\right|_{c_{T} k=a H},
\end{aligned}
$$



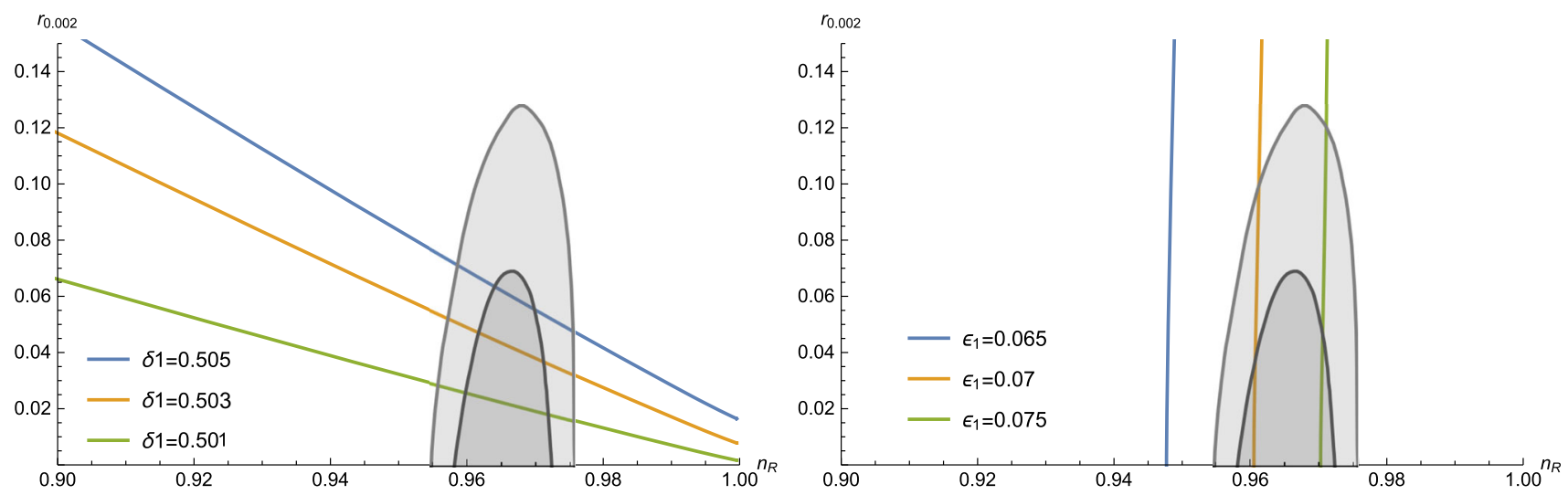

Fig. 9 The $n_{\mathcal{R}}-r$ region predicted by the model for the parameter region III of Fig. 6

with the scalar spectral index and the tensor-to-scalar ratio are

$$
\begin{aligned}
& n_{\mathcal{R}}-1=3-2 v_{\mathcal{R}}, \\
& r \equiv \frac{\mathcal{P}_{T}}{\mathcal{P}_{\mathcal{R}}} \simeq 2^{3+2 v_{T}-2 v_{\mathcal{R}}} F \frac{c_{\mathcal{R}}^{3}}{c_{T}^{3}} \frac{\Gamma^{2}\left(v_{T}\right)}{\Gamma^{2}\left(v_{\mathcal{R}}\right)} \frac{\left(1+\frac{\epsilon_{1}}{1+2 \eta_{H}}\right)^{2 v_{\mathcal{R}}-2 v_{T}}}{(1-\Delta / 2)^{2}\left(1-\delta_{1}\right)} .
\end{aligned}
$$

Similarly as in the previous section, we set the parameter $\delta_{1}$ to be a constant and discuss two cases: $\delta_{1}=0$ or $\delta \neq 0$.

\section{$5.1 \delta_{1}=0$}

If $\delta_{1}=0$ and $\eta_{H}=$ constant, which is the original constantroll model without GB coupling [3]. In this case, to the first

$n_{\mathcal{R}} \simeq 1-\frac{2\left(2 \eta_{H}-3\right)\left(4 \eta_{H}+8 \eta_{H}^{2}+\delta_{1}\left(2-6 \eta_{H}-12 \eta_{H}^{2}\right)+\delta_{1}^{2}\left(-5+2 \eta_{H}+4 \eta_{H}^{2}\right)\right)}{3 \delta_{1}\left(5 \delta_{1}-2\right)\left(1+2 \eta_{H}\right)} \epsilon_{1}$,

If $\delta_{1}=$ constant but not zero, the scalar spectral index and the tensor-to-scalar ratio to the first order of $\epsilon_{1}$ is obtained as

Then we get the $n_{\mathcal{R}}-r$ predictions with $N=60$ and show them in Fig. 5 (orange line). For a constant $\eta_{H}$, the result is consistent with the observations at $2 \sigma$ confidence level. And we updated the constraint to $\eta_{H}$ with the Planck 2018 data, which is $-0.0163<\eta_{H}<-0.0006$.

\section{$5.2 \delta_{1} \neq 0$} Since $\eta_{H}$ is a constant, from the definition of flow parameters (5) and (6), and the condition $\epsilon_{1}(N=0)=1$, we obtain the relation [18]

$\epsilon_{1}(N)=\frac{\eta_{H} \exp \left(2 \eta_{H} N\right)}{\exp \left(2 \eta_{H} N\right)+\eta_{H}-1}$.

and

order approximation of $\epsilon_{1}$, the scalar spectral index can be approximated as

$n_{\mathcal{R}} \simeq 4-\left|2 \eta_{H}-3\right|+\frac{2\left(4 \eta_{H}^{2}+5 \eta_{H}-6\right) \epsilon_{1}}{\left|2 \eta_{H}-3\right|\left(2 \eta_{H}+1\right)}$

and the tensor-to-scalar ratio

$r \simeq 2^{3-\left|3-2 \eta_{H}\right|}\left(\frac{\Gamma[3 / 2]}{\Gamma\left[\left|3-2 \eta_{H}\right| / 2\right]}\right)^{2} 16 \epsilon_{1}$

$$
\begin{aligned}
r \simeq & -\frac{16}{3\left(3 \delta_{1}-2\right)\left(5 \delta_{1}-2\right)} \sqrt{\frac{2-7 \delta_{1}+6 \delta_{1}^{2}}{2-5 \delta_{1}}}\left(30 \delta_{1}^{3}-27 \delta_{1}^{2}\right. \\
& +6 \delta_{1}+\epsilon_{1}\left(45 \delta_{1}^{4}-69 \delta_{1}^{3}-6 \delta_{1}^{2}+42 \delta_{1}-12\right. \\
& +2\left(-2 \delta_{1}^{3}+7 \delta_{1}^{2}-7 \delta_{1}+2\right) \eta_{H}\left(2 \eta_{H}-3\right) \ln 4 \\
& \left.\left.+4\left(2 \delta_{1}^{3}-7 \delta_{1}^{2}+7 \delta_{1}-2\right) \eta_{H}\left(2 \eta_{H}-3\right)(\gamma-2+\ln 4)\right)\right) .
\end{aligned}
$$




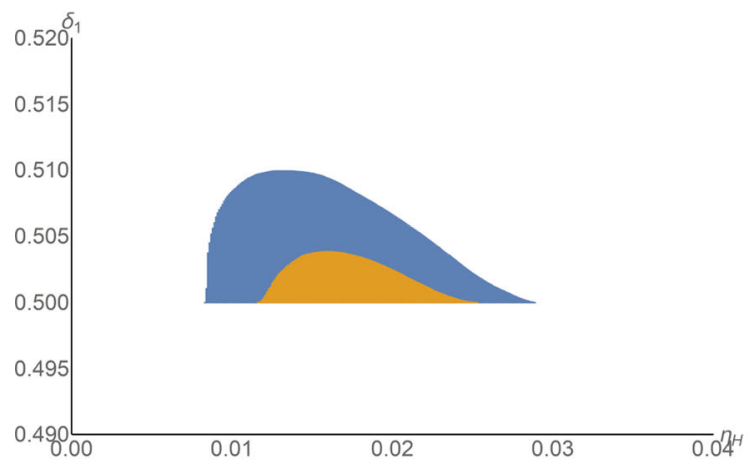

Region I

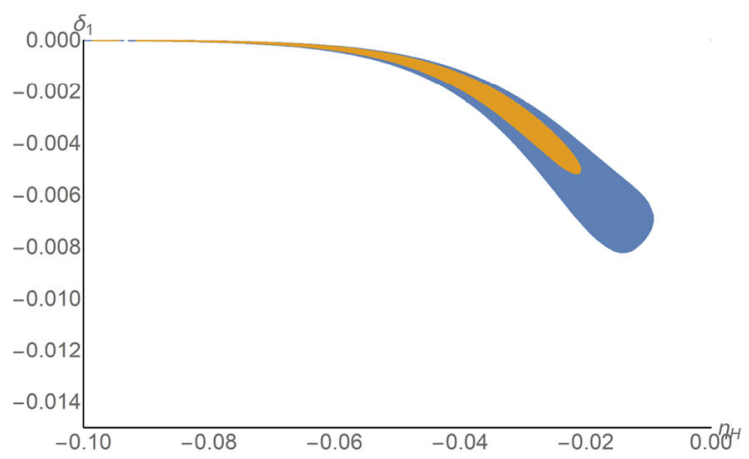

Region III

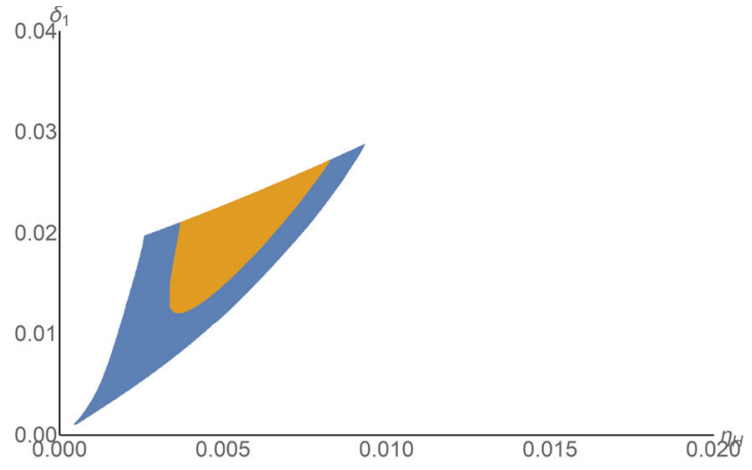

Region II

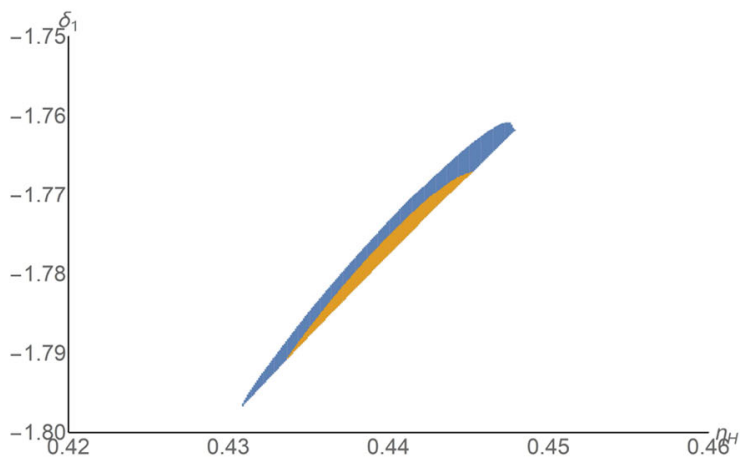

Region IV

Fig. 10 The observational constraints on $\eta_{H}$ and $\delta_{1}$ with the e-folding number $N=60$. The orange and blue regions correspond to the parameters satisfied $1 \sigma$ and $2 \sigma$ confidence level, respectively

Using the relation (48) and setting the e-folds $N=60$, we find four reasonable regions of parameter space, which are show in Fig. 10. We can see that in this case of constant-roll inflation, the absolute of GB flow parameter $\delta_{1}$ can be larger then one(region IV), which is different from the conventional slow-roll inflation with GB coupling.

The tensor-to-scalar ratio $r$ versus the spectral index $n_{\mathcal{R}}$ for the parameters region I of Fig. 10 are show in Fig. 11. $\delta_{1}$ is taken as $\delta_{1}=0.502,0.506,0.51$ from left to right in the left panel, and the $n_{\mathcal{R}}-r$ dots go along the curves from right to left as $\eta_{H}$ increase. The parameters $\eta_{H}$ taken as $\eta_{H}=0.03,0.02,0.01$ are show in the right panel, and this time the $n_{\mathcal{R}}-r$ dots go from bottom to top as $\delta_{1}$ increase.

In Fig. 12, we show $n_{\mathcal{R}}$ versus $r$ for the parameters in region II of Fig. 10. Left panel: The parameter $\delta_{1}$ is taken as $\delta_{1}=0.025,0.015,0.005$ from left to right, and as $\eta_{H}$ increase, the $n_{\mathcal{R}}-r$ dots go along the curves from right to left. Right panel: The parameters $\eta_{H}$ is taken as $\eta_{H}=$ $0.009,0.005,0.001$ from left to right, and as $\delta_{1}$ increase, the $n_{\mathcal{R}}-r$ dots go along the curves from left to right.Left panel: The parameter $\delta_{1}$ is taken as $\delta_{1}=0.025,0.015,0.005$ from left to right, and as $\eta_{H}$ increase, the $n_{\mathcal{R}}-r$ dots go along the curves from right to left. Right panel: The parameters $\eta_{H}$ is taken as $\eta_{H}=0.009,0.005,0.001$ from left to right, and as $\delta_{1}$ increase, the $n_{\mathcal{R}}-r$ dots go along the curves from left to right.

Similarly, the $n_{\mathcal{R}}$ versus $r$ for the parameters in region III of Fig. 10 are show in Fig. 13. In the left panel The parameter is taken as $\delta_{1}=-0.002,-0.005,-0.008$ from left to right, and the dots go along the curves to the top as $\eta_{H}$ increase. The parameter $\eta_{H}$ is taken as $\eta_{H}=-0.01,-0.03,-0.05$ from left to right in the right panel. In this time the $n_{\mathcal{R}}-r$ dots go from left to right as $\delta_{1}$ increase.

Finally, the $n_{\mathcal{R}}-r$ constraint for the parameters in region IV of Fig. 10 are show in Fig. 14 with the parameters taken as $\delta_{1}=-1.8,-1.78,-1.76$ from left to right in the left, where the $n_{\mathcal{R}}-r$ dots go from bottom to top as $\eta_{H}$ increase. The parameter in the right panels $\eta_{H}=0.43,0.44,0.45$ from left to right. In this time as $\delta_{1}$ increase, the dots go along the curves from bottom to top. 

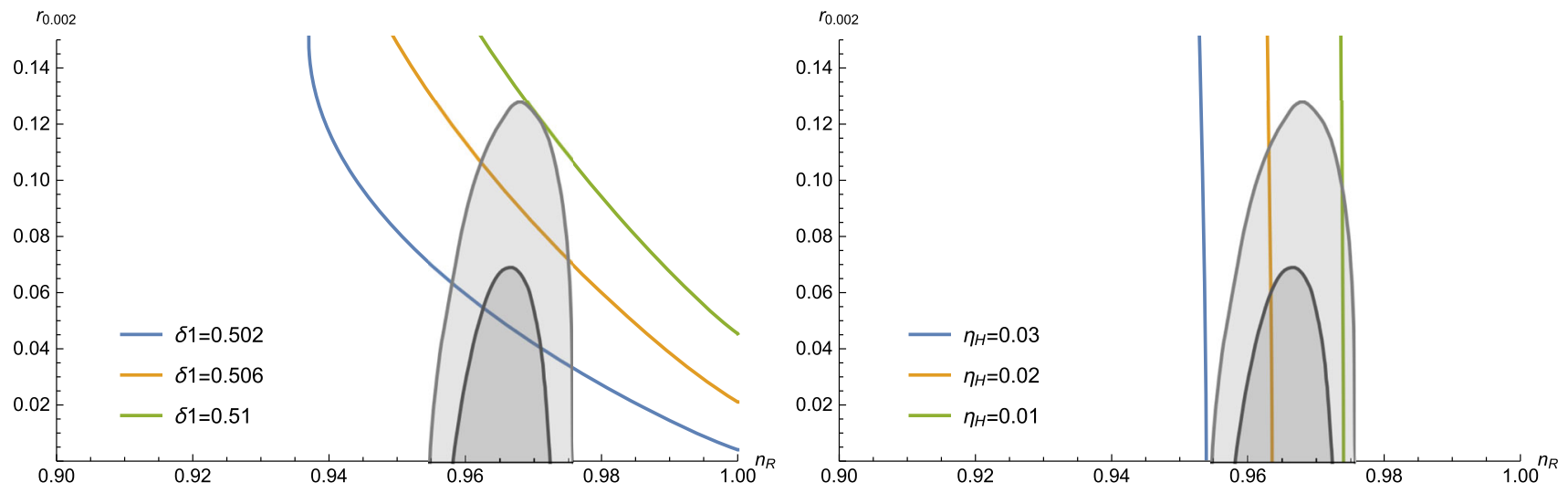

Fig. 11 The $n_{\mathcal{R}}-r$ region predicted by the model for the parameter region I of Fig. 10
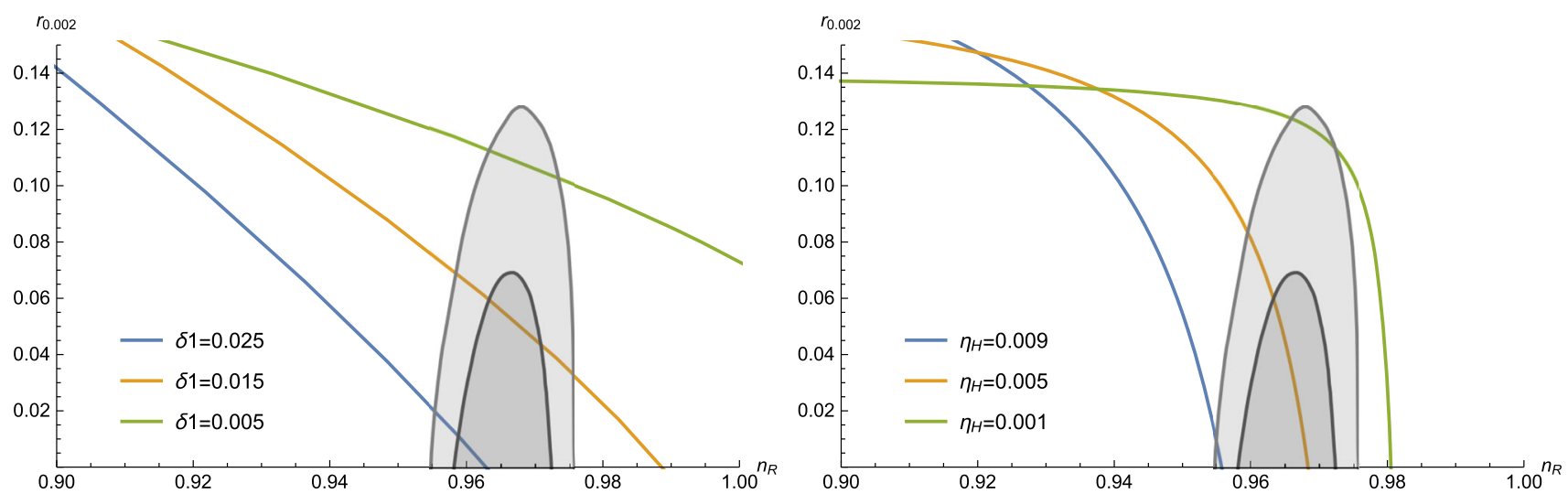

Fig. 12 The $n_{\mathcal{R}}-r$ region predicted by the model for the parameter region II of Fig. 10
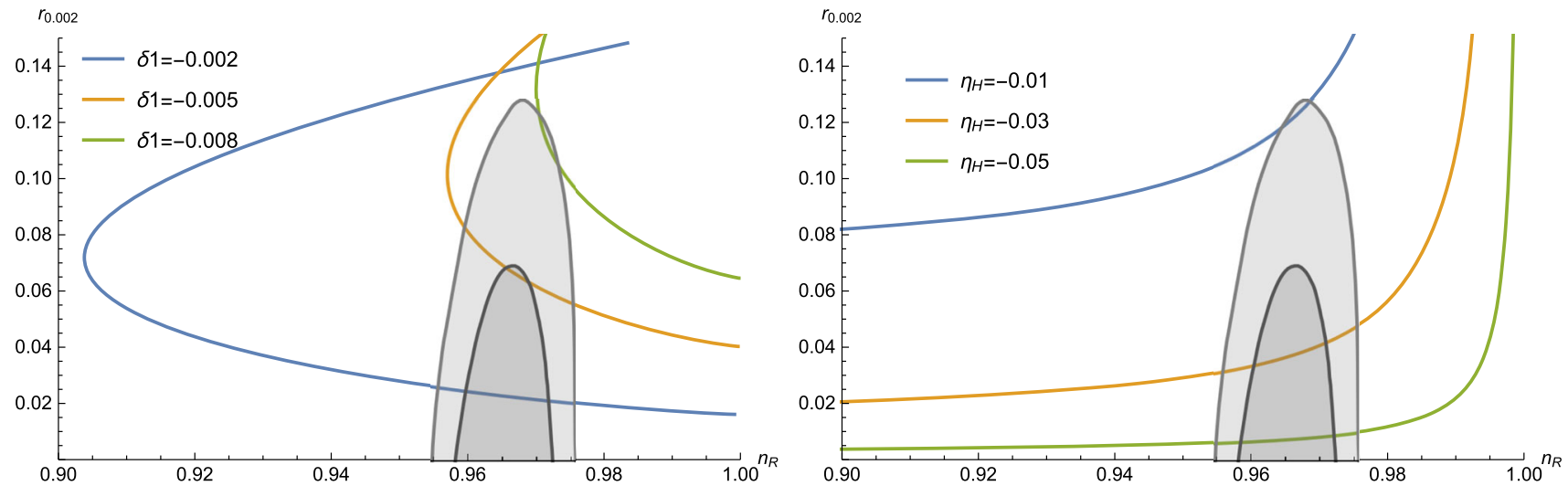

Fig. 13 The $n_{\mathcal{R}}-r$ region predicted by the model for the parameter region III of Fig. 10

\section{Reconstruct the potential}

In this section, we shall find the corresponding scalar potential $V(\phi)$ and the GB coupling $\xi(\phi)$ for the constant-roll inflation. If the GB flow parameter $\delta_{1}$ is a constant, from the definition of flow parameters, we have $\delta_{2}=0$, then the background equations (2) and (3) can be written as

$$
\omega \dot{\phi}^{2}=\left(2 \epsilon_{1}-\delta_{1}-\delta_{1} \epsilon_{1}\right) H^{2},
$$

$$
6\left(1-\delta_{1}\right) H^{2}=\omega \dot{\phi}^{2}+2 V .
$$

In the following, we will discuss the models with $\epsilon_{1}=$ constant, $\epsilon_{2}=$ constant and $\eta_{H}=$ constant, respectively.

$6.1 \epsilon_{1}=$ constant

In the model with $\epsilon_{1}=$ constant, the case with $\delta_{1}=0$ is ruled out by the observations, so we only interest in the case with 

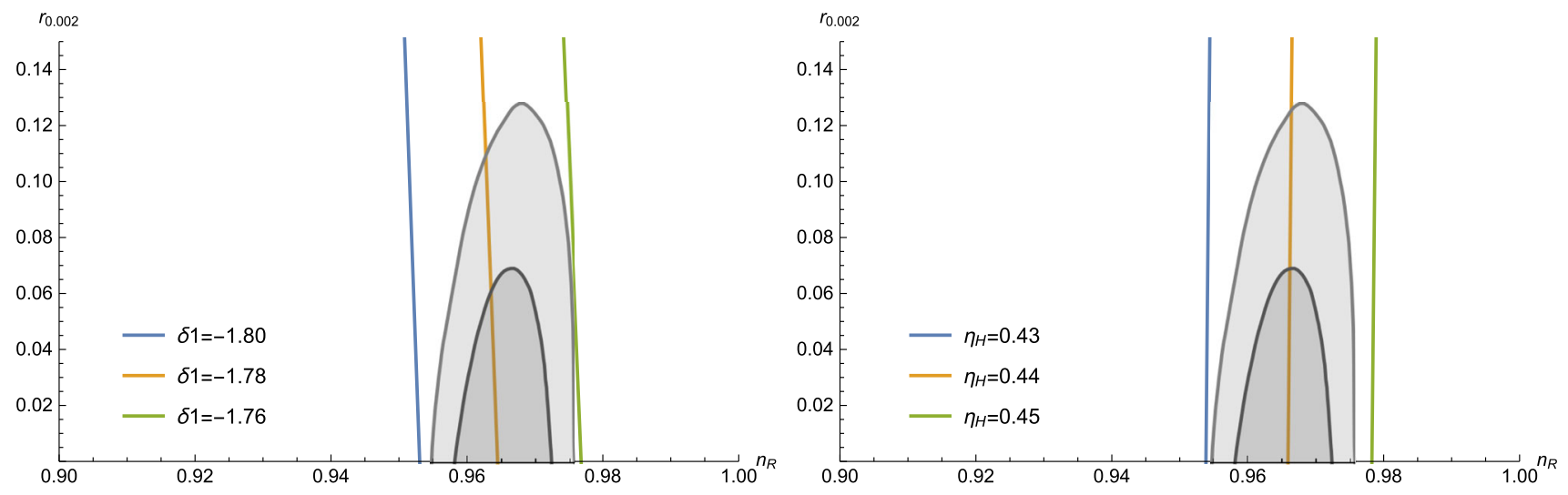

Fig. 14 The $n_{\mathcal{R}}-r$ region predicted by the model for the parameter region IV of Fig. 10

$\delta_{1}=$ constant but not zero. Substitute (51) into the definition of the flow parameter $\epsilon_{1}$

$\epsilon_{1}=-\frac{\dot{H}}{H^{2}}=-\frac{\dot{\phi} H_{, \phi}}{H^{2}}$,

we obtain a first-order differential equation of the Hubble parameter and get two solutions

$H(\phi)=c_{1} e^{ \pm \frac{i \epsilon 1 \sqrt{\omega} \phi}{\sqrt{\delta_{1}-2 \epsilon_{1}+\delta_{1} \epsilon_{1}}}}$,

with $c_{1}$ is a integration constant. Substitute the Eqs. (54) and (51) into the Hamilton-Jacobi equation (52), we get the potential of the scalar field $\phi$

$V(\phi)=V_{0}\left(6-5 \delta_{1}-2 \epsilon_{1}+\delta_{1} \epsilon_{1}\right) e^{ \pm \frac{2 i \epsilon_{1} \sqrt{\omega} \phi}{\sqrt{\delta_{1}-2 \epsilon_{1}+\delta_{1} \epsilon_{1}}}}$

where the overall factor $V_{0}=c_{1}^{2} / 2$ can be restricted by the amplitude of the primordial curvature perturbations.

For the GB coupling $\xi(\phi)$, we substitute the result of Hubble parameter (54) into the definition of GB flow parameter $\delta_{1}=4 \dot{\xi} H$ and get a differential equation of $\xi(\phi)$, the solutions are

$\xi(\phi)=\frac{\delta_{1}}{16 \epsilon_{1} V_{0}} e^{\mp \frac{2 i \epsilon_{1} \sqrt{\omega} \phi}{\sqrt{\delta_{1}-2 \epsilon_{1}+\delta_{1} \epsilon_{1}}}}+\xi_{0}$

where $\xi_{0}$ is an integration constant represents the GB term with out coupling to $\phi$, which is no contribution to the result, and is always set to zero. In addition, the coefficient $\omega$ in the expressions can be chosen the values \pm 1 to ensure the results $H(\phi), V(\phi), \xi(\phi)$ are real functions and generate reasonable inflation. For instance, if $\delta_{1}-2 \epsilon_{1}+\delta_{1} \epsilon_{1}>0, \omega$ should be chosen -1 .
$6.2 \epsilon_{2}=$ constant

If the flow parameter $\epsilon_{2}$ is a constant, as discussed in Sect. 3, the case with $\delta_{1}=0$ is also ruled out by the observations. For the case with $\delta_{1} \neq 0$, the differential equation to the Hubble parameter is complicated and no analytic solutions. However, for some special cases, we can get the approximate expressions. In the following, we focus on two special cases: $\epsilon_{1} \ll \delta_{1}$ and $\delta_{1} \ll \epsilon_{1}<1$.

\section{$6.2 .1 \epsilon_{1} \ll \delta_{1}$}

We first discuss the relation $\epsilon_{1} \ll \delta_{1}$, which can be satisfied near the beginning of inflation. In this case, the background equation (51) is approximated as

$\omega \dot{\phi}^{2}=-\delta_{1} H^{2}$

Substitute it into the definition of the flow parameter $\epsilon_{2}$

$$
\epsilon_{2} \equiv \frac{\dot{\epsilon}_{1}}{H \epsilon_{1}}=\frac{\dot{\phi}^{2} H_{, \phi \phi}+\ddot{\phi} H_{, \phi}}{\dot{\phi} H H_{\phi}}-\frac{2 \dot{\phi} H_{, \phi}}{H^{2}},
$$

and solve the second-order differential equation of $H$, we get the Hubble parameter as a function of $\phi$

$H(\phi)=c_{2} e^{ \pm \frac{i c_{1} \sqrt{\delta_{1}} \exp \left[\mp \frac{i \epsilon_{2} \sqrt{\omega} \phi}{\sqrt{\delta_{1}}}\right]}{\epsilon_{2} \sqrt{\omega}}}$,

with $c_{1}$ and $c_{2}$ are integration constants. Then combine the solution (59) with the background equations (51) and (52), we get the potential of the scalar field $\phi$

$V(\phi)=V_{0}\left(6-5 \delta_{1}\right) e^{ \pm \frac{2 i c_{1} \sqrt{\delta_{1}} \exp \left[\mp \frac{i \epsilon_{2} \sqrt{\omega} \phi}{\sqrt{\delta_{1}}}\right]}{\epsilon_{2} \sqrt{\omega}}}$,

where $V_{0}=c_{2}^{2} / 2$ is the overall factor. 
Combine of the definition $\delta_{1}=4 \dot{\xi} H$ and the Hubble parameter (59), we get a differential equation the GB coupling $\xi(\phi)$, and the solutions are

$\xi(\phi)=\frac{\delta 1}{8 \epsilon_{2} V_{0}} \operatorname{Ei}\left(\mp \frac{2 i c_{1} \sqrt{\delta_{1}} \exp \left[\mp \frac{i \epsilon_{2} \sqrt{\omega} \phi}{\sqrt{\delta_{1}}}\right]}{\epsilon_{2} \sqrt{\omega}}\right)+\xi_{0}$,

where $E i(x) \equiv-\int_{-x}^{\infty} \frac{e^{-t}}{t} d t$ is the exponential integral function, and $\xi_{0}$ is a integration constant represent the GB term with out coupling to $\phi$.

\subsection{2 $\delta_{1} \ll \epsilon_{1}$}

The relation $\delta_{1} \ll \epsilon_{1}<1$ is likely to happen near the end of inflation, then the background equation (51) is approximated as

$\omega \dot{\phi}^{2}=2 \epsilon_{1} H^{2}$,

combine with the definition of $\epsilon_{1}$, we get the relation

$\omega \dot{\phi}=-2 H_{, \phi}$,

which is the same as the standard slow-roll inflation without GB coupling. Substitute (63) into the definition of the flow parameter $\epsilon_{2}$, Eq. (58), we obtain the differential equation of the Hubble parameter and the solution is

$H(\phi)=c_{2} e^{c_{1} \phi-\frac{1}{8} \epsilon_{2} \omega \phi^{2}}$,

with $c_{1}$ and $c_{2}$ are integration constants. The potential of the scalar field can be obtained by substitute the Eqs. (63) and (64) into (52), which can be written as

$V(\phi)=V_{0}\left(6-6 \delta_{1}-\frac{4 c_{1}^{2}}{\omega}+2 c_{1} \epsilon_{2} \phi-\frac{1}{4} \epsilon_{2}^{2} \omega \phi^{2}\right) e^{2 c_{1} \phi-\frac{1}{4} \epsilon_{2} \omega \phi^{2}}$,

with the overall factor $V_{0}=c_{2}^{2} / 2$.

Substitute the result of Hubble parameter (64) into the definition of GB flow parameter $\delta_{1}=4 \dot{\xi} H$, then we get the solution of GB coupling

$\xi(\phi)=\frac{\delta_{1}}{8 \epsilon_{2} V_{0}} e^{-\frac{4 c_{1}^{2}}{\epsilon_{2} \omega}} \mathrm{Ei}\left[\frac{\left(\epsilon_{2} \omega \phi-4 c_{1}\right)^{2}}{4 \epsilon_{2} \omega}\right]+\xi_{0}$.

\section{$6.3 \eta_{H}=$ constant}

In this section, we reconstruct the potential for the constantroll inflation with $\eta_{H}$ and $\delta_{1}$ are constants. If $\delta_{1}=0$, the model recover to the case without GB coupling, which is discussed in other reference [3]. Form the background equation, we get the relation

$\omega \dot{\phi}=-2 H_{, \phi}$

Using the same method as in the previous subsections and combine the definition of $\eta_{H}$

$\eta_{H} \equiv-\frac{\ddot{H}}{2 H \dot{H}}=-\frac{\dot{\phi}^{2} H_{, \phi \phi}+\ddot{\phi} H_{, \phi}}{2 \dot{\phi} H H_{\phi}}$,

we get the Hubble parameter and the scalar potential as functions of $\phi$

$$
\begin{aligned}
& H(\phi)=c_{1} e^{\frac{\sqrt{\eta_{H} \sqrt{\omega} \phi}}{\sqrt{2}}}+c_{2} e^{-\frac{\sqrt{\eta_{H}} \sqrt{\omega} \phi}{\sqrt{2}}}, \\
& V(\phi)=2 c_{1} c_{2}\left(3+\eta_{H}\right)+\left(3-\eta_{H}\right)\left(c_{1}^{2} e^{\sqrt{2 \eta_{H}} \sqrt{\omega} \phi}+c_{2}^{2} e^{-\sqrt{2 \eta_{H}} \sqrt{\omega} \phi}\right),
\end{aligned}
$$

with $c_{1}$ and $c_{2}$ are integration constants. The results are consistent with other reference [3].

Similarly, for the case with $\delta_{1} \neq 0$, the differential equation to the Hubble parameter is no analytic solutions. And we discuss the approximate expressions in two special cases: $\epsilon_{1} \ll \delta_{1}$ and $\delta_{1} \ll \epsilon_{1}<1$.

\subsection{1 $\epsilon_{1} \ll \delta_{1}$}

If $\epsilon_{1} \ll \delta_{1}$, combine the background equations (57) and (52) with the definition of parameter $\eta_{H}(68)$, we can get two solutions of the Hubble parameter $H(\phi)$ and the corresponding scalar potential $V(\phi)$

$$
\begin{aligned}
& H(\phi)=c_{2}\left(e^{ \pm \frac{2 i c_{1} \eta_{H} \sqrt{\omega}}{\sqrt{\delta_{1}}}}-e^{ \pm \frac{2 i \eta_{H} \sqrt{\omega} \phi}{\sqrt{\delta_{1}}}}\right)^{\frac{1}{2}}, \\
& V(\phi)=V_{0}\left(6-5 \delta_{1}\right)\left(e^{ \pm \frac{2 i c_{1} \eta_{H} \sqrt{\omega}}{\sqrt{\delta_{1}}}}-e^{ \pm \frac{2 i \eta_{H} \sqrt{\omega} \phi}{\sqrt{\delta_{1}}}}\right),
\end{aligned}
$$

with $c_{1}$ and $c_{2}$ are integration constants and $V_{0}=c_{2}^{2} / 2$. Substitute the Hubble parameter (71) into the definition of GB flow parameter $\delta_{1}=4 \dot{\xi} H$, then the solution of GB coupling can be written as

$\xi(\phi)=\xi_{0}+\frac{\delta_{1}}{16 V_{0} \eta_{H}} e^{\frac{2 i c_{1} \eta_{H} \sqrt{\omega}}{\sqrt{\delta_{1}}}} \ln \left[e^{\frac{2 i c_{1} \eta_{H} \sqrt{\omega}}{\sqrt{\delta_{1}}}}-e^{\frac{2 i \eta_{H} \sqrt{\omega} \phi}{\sqrt{\delta_{1}}}}\right]$,

for the + case of (71), and for the - case is

$$
\begin{aligned}
\xi(\phi)= & \xi_{0}+\frac{\delta_{1}}{16 V_{0} \eta_{H}} e^{-\frac{2 i c_{1} \eta_{H} \sqrt{\omega}}{\sqrt{\delta_{1}}}}\left(\ln \left[e^{\frac{2 i c_{1} \eta_{H} \sqrt{\omega}}{\sqrt{\delta_{1}}}}-e^{\frac{2 i \eta_{H} \sqrt{\omega} \phi}{\sqrt{\delta_{1}}}}\right]\right. \\
& \left.-\frac{2 i \eta_{H} \sqrt{\omega} \phi}{\sqrt{\delta_{1}}}\right) .
\end{aligned}
$$




\section{$6.3 .2 \delta_{1} \ll \epsilon_{1}$}

If $\delta_{1} \ll \epsilon_{1}<1$, using the same method, combine the relation (63) with the definition of parameter $\eta_{H}$ (68), we can get the solutions of the Hubble parameter $H(\phi)$

$H(\phi)=c_{1} e^{\frac{\sqrt{\eta_{H}} \sqrt{\omega} \phi}{\sqrt{2}}}+c_{2} e^{-\frac{\sqrt{\eta_{H}} \sqrt{\omega} \phi}{\sqrt{2}}}$,

then substitute it into the background equation (52), we get the scalar potential $V(\phi)$

$$
\begin{aligned}
V(\phi)= & 2 c_{1} c_{2}\left(3+\eta_{H}-3 \delta_{1}\right)+\left(3-\eta_{H}-3 \delta_{1}\right) \\
& \times\left(c_{1}^{2} e^{\sqrt{2 \eta_{H}} \sqrt{\omega} \phi}+c_{2}^{2} e^{-\sqrt{2 \eta_{H}} \sqrt{\omega} \phi}\right),
\end{aligned}
$$

and the GB coupling $\xi(\phi)$ can be obtained by integer the definition of $\delta_{1}$

$\xi(\phi)=\frac{\delta_{1}}{8 c_{1} c_{2} \eta_{H}} \tanh ^{-1}\left[\frac{c_{1} \mathrm{e}^{\sqrt{2 \eta_{H}} \sqrt{\omega} \phi}}{c_{2}}\right]+\xi_{0}$.

\section{Summary}

In this paper, we discuss the constant-roll inflation in the model with the inflaton $\phi$ nonminimal coupled to the GB term. Since the presence of a new degree of freedom from GB coupling $\xi(\phi)$, so we discuss the constant-roll inflation with constant $\epsilon_{1}$, constant $\epsilon_{2}$ and constant $\eta_{H}$, with the additional assumption that the first GB flow parameter $\delta_{1}$ is a constant, respectively. Using the Bessel function approximation, we present the mode equations of scalar and tensor perturbations and get the analytical expressions for the scalar and tensor power spectrum to the first order of $\epsilon_{1}$. We derive the scalar spectral index and the tensor to scalar ratio and constraint the parameter space by using the Planck 2018 results. First, we assume that $\epsilon_{1}$ is a constant, the case with $\delta_{1}=0$ is ruled out by the observations, and in the case with $\delta_{1}=$ constant but not zero, we obtain two regions of parameter space and show the results on the $n_{\mathcal{R}}-r$ region. Second, in the assumption with $\epsilon_{2}=$ constant, $\delta_{1}=0$ is just the constant-roll inflation without GB coupling, which is also ruled out by the observations. If $\delta_{1}=$ constant but not zero, we obtain three regions of parameter space. Finally, we assume that $\eta_{H}=$ constant, the case with $\delta_{1}=0$ is the original constant-roll inflation discussed in [3]. Here we update the constraint from Planck 2018 data, which is $-0.0163<\eta_{H}<-0.0006$. In the case with $\delta_{1}=$ constant but not zero, we obtain four feasible regions of parameter space, and find that in some region, the chosen of GB flow parameter $\delta_{1}>1$ can also produce a nearly scale-invariant scalar power spectrum, which is different from the conventional slow-roll inflation with GB coupling. In addition, we also find the analytical expressions for corresponding scalar potential of the constant-roll inflation and the nonminimum coupling $\xi(\phi)$ in some cases.

Acknowledgements We would like to thank Zong-Kuan Guo for useful conversations. This work was supported by "the National Natural Science Foundation of China” (NNSFC) with Grant no. 11705133.

Data Availability Statement This manuscript has no associated data or the data will not be deposited. [Authors' comment: This is a theoretical study and the results can be obtained explicitly from the relevant calculations presented in this paper.]

Open Access This article is licensed under a Creative Commons Attribution 4.0 International License, which permits use, sharing, adaptation, distribution and reproduction in any medium or format, as long as you give appropriate credit to the original author(s) and the source, provide a link to the Creative Commons licence, and indicate if changes were made. The images or other third party material in this article are included in the article's Creative Commons licence, unless indicated otherwise in a credit line to the material. If material is not included in the article's Creative Commons licence and your intended use is not permitted by statutory regulation or exceeds the permitted use, you will need to obtain permission directly from the copyright holder. To view a copy of this licence, visit http://creativecomm ons.org/licenses/by/4.0/.

Funded by SCOAP ${ }^{3}$.

\section{References}

1. G. Hinshaw et al., WMAP. Astrophys. Nine-Year Wilkinson Microwave Anisotropy Probe (WMAP) observations: Cosmological parameter results. J. Suppl. 208, 19 (2013). arXiv:1212.5226 [astro-ph.CO]

2. Y. Akrami et al. [Planck], Planck 2018 results. X. Constraints on inflation. arXiv:1807.06211 [astro-ph.CO]

3. H. Motohashi, A.A. Starobinsky, J. Yokoyama, Inflation with a constant rate of roll. JCAP 09, 018 (2015). arXiv:1411.5021 [astroph.CO]

4. N.C. Tsamis, R.P. Woodard, Improved estimates of cosmological perturbations. Phys. Rev. D 69, 084005 (2004). arXiv:astro-ph/0307463

5. W.H. Kinney, Horizon crossing and inflation with large eta. Phys. Rev. D 72, 023515 (2005). arXiv:gr-qc/0503017

6. H. Motohashi, A.A. Starobinsky, Constant-roll inflation: confrontation with recent observational data. EPL 117(3), 39001 (2017). arXiv:1702.05847 [astro-ph.CO]

7. S.D. Odintsov, V.K. Oikonomou, Inflationary dynamics with a smooth slow-roll to constant-roll era transition. JCAP 04, 041 (2017). arXiv:1703.02853 [gr-qc]

8. L. Anguelova, P. Suranyi, L.C.R. Wijewardhana, Systematics of constant roll inflation. JCAP 02, 004 (2018). arXiv:1710.06989 [hep-th]

9. S. Nojiri, S.D. Odintsov, V.K. Oikonomou, Constant-roll inflation in $F(R)$ gravity. Class. Quantum Gravity 34(24), 245012 (2017). arXiv: 1704.05945 [gr-qc]

10. Z. Yi, Y. Gong, M. Sabir, Inflation with Gauss-Bonnet coupling. Phys. Rev. D 98(8), 083521 (2018). arXiv:1804.09116 [gr-qc]

11. H. Motohashi, A.A. Starobinsky, Constant-roll inflation in scalartensor gravity. JCAP 11, 025 (2019). arXiv:1909.10883 [gr-qc]

12. S.D. Odintsov, V.K. Oikonomou, Constant-roll $k$-inflation dynamics. Class. Quantum Gravity 37(2), 025003 (2020). arXiv:1912.00475 [gr-qc] 
13. I. Antoniadis, A. Lykkas, K. Tamvakis, Constant-roll in the Palatini- $R^{2}$ models. JCAP 04(04), 033 (2020). arXiv:2002.12681 [gr-qc]

14. A. Mohammadi, T. Golanbari, S. Nasri, K. Saaidi, Constantroll brane inflation. Phys. Rev. D 101(12), 123537 (2020). arXiv:2004.12137 [gr-qc]

15. A. Micu, Two-field constant roll inflation. JCAP 11, 003 (2019). arXiv:1904.10241 [hep-th]

16. Q. Gao, The observational constraint on constant-roll inflation. Sci. China Phys. Mech. Astron. 61(7), 070411 (2018). arXiv: 1802.01986 [gr-qc]

17. H. Motohashi, A.A. Starobinsky, $f(R)$ constant-roll inflation. Eur. Phys. J. C 77(8), 538 (2017). arXiv:1704.08188 [astro-ph.CO]

18. Q. Gao, Y. Gong, Q. Fei, Constant-roll tachyon inflation and observational constraints. JCAP 05, 005 (2018). arXiv:1801.09208 [grqc]

19. Q. Gao, Reconstruction of constant slow-roll inflation. Sci. China Phys. Mech. Astron. 60(9), 090411 (2017). arXiv:1704.08559 [astro-ph.CO]

20. Z. Yi, Y. Gong, On the constant-roll inflation. JCAP 03, 052 (2018). arXiv: 1712.07478 [gr-qc]

21. H. Motohashi, S. Mukohyama, M. Oliosi, Constant roll and primordial blackholes. JCAP 03, 002 (2020). arXiv:1910.13235 [gr-qc]

22. A. Oliveros, H.E. Noriega, Constant-roll inflation driven by a scalar field with nonminimal derivative coupling. Int. J. Mod. Phys. D 28(12), 1950159 (2019). arXiv:1907.10694 [gr-qc]

23. D.J. Grossa, J.H. Sloana, The quartic effective action for the heterotic string. Nucl. Phys. B 291, 41 (1987)

24. M. Gasperini, M. Maggiore, G. Veneziano, Towards a nonsingular pre-big bang cosmology. Nucl. Phys. B 494, 315330 (1997). https://doi.org/10.1016/S0550-3213(97)00149-1. arXiv:hep-th/9611039

25. K. Bamba, Z.K. Guo, N. Ohta, Accelerating cosmologies in the Einstein-Gauss-Bonnet theory with dilaton. Prog. Theor. Phys. 118, 879-892 (2007). arXiv:0707.4334 [hep-th]

26. M. Satoh, S. Kanno, J. Soda, Circular polarization of primordialgravitational waves in string-inspired inflationary cosmology. Phys. Rev. D 77, 023526 (2008). arXiv:0706.3585 [astro-ph]

27. S. Nojiri, S.D. Odintsov, M. Sasaki, Gauss-Bonnet dark energy. Phys. Rev. D 71, 123509 (2005). arXiv:hep-th/0504052

28. J.H. He, B. Wang, E. Papantonopoulos, Observational constrains on the DGP brane-world model with a Gauss-Bonnet term in the bulk. Phys. Lett. B 654, 133-138 (2007). arXiv:0707.1180 [gr-qc]
29. Z.K. Guo, D.J. Schwarz, Power spectra from an inflaton coupled to the Gauss-Bonnet term. Phys. Rev. D 80, 063523 (2009). arXiv:0907.0427 [hep-th]

30. Z.K. Guo, D.J. Schwarz, Slow-roll inflation with a Gauss-Bonnet correction. Phys. Rev. D 81, 123520 (2010). arXiv:1001.1897 [hepth]

31. P.X. Jiang, J.W. Hu, Z.K. Guo, Inflation coupled to a Gauss-Bonnet term. Phys. Rev. D 88, 123508 (2013). arXiv:1310.5579 [hep-th]

32. E.O. Pozdeeva, M.R. Gangopadhyay, M. Sami, A.V. Toporensky, S.Y. Vernov, Inflation with a quartic potential in the framework of Einstein-Gauss-Bonnet gravity. arXiv:2006.08027 [gr-qc]

33. E.O. Pozdeeva, Generalization of cosmological attractor approach to Einstein-Gauss-Bonnet gravity. Eur. Phys. J. C 80(7), 612 (2020). arXiv:2005.10133 [gr-qc]

34. E.O. Pozdeeva, M. Sami, A.V. Toporensky, S.Y. Vernov, Stability analysis of de Sitter solutions in models with the Gauss-Bonnet term. Phys. Rev. D 100(8), 083527 (2019). arXiv:1905.05085 [grqc]

35. S.D. Odintsov, V.K. Oikonomou, F.P. Fronimos, Non-minimally coupled Einstein Gauss Bonnet inflation phenomenology in view of GW170817. arXiv:2007.02309 [gr-qc]

36. S. Odintsov, V. Oikonomou, Swampland implications of GW170817-compatible Einstein-Gauss-Bonnet gravity. Phys. Lett. B 805, 135437 (2020). arXiv:2004.00479 [gr-qc]

37. V. Oikonomou, F. Fronimos, Reviving non-Minimal Horndeskilike Theories after GW170817: Kinetic coupling corrected Einstein-Gauss-Bonnet inflation. arXiv:2006.05512 [gr-qc]

38. J. Mathew, S. Shankaranarayanan, Low scale Higgs inflation with Gauss-Bonnet coupling. Astropart. Phys. 84, 1-7 (2016). arXiv:1602.00411 [astro-ph.CO]

39. S.D. Odintsov, V.K. Oikonomou, Viable Inflation in Scalar-GaussBonnet Gravity and Reconstruction from Observational Indices. Phys. Rev. D 98(4), 044039 (2018). arXiv:1808.05045 [gr-qc]

40. C. van de Bruck, C. Longden, Higgs Inflation with a Gauss-Bonnet term in the Jordan Frame. Phys. Rev. D 93(6), 063519 (2016). arXiv:1512.04768 [hep-ph]

41. D. Samart, P. Channuie, Unification of inflation and dark matter in the Higgs-Starobinsky model. Eur. Phys. J. C 79(4), 347 (2019). arXiv: 1812.11180 [gr-qc]

42. L.N. Granda, D.F. Jimenez, Slow-Roll Inflation in Scalar-Tensor Models. JCAP 09, 007 (2019). arXiv:1905.08349 [gr-qc] 